Herzgesund zur Arbeit mit dem Bus?

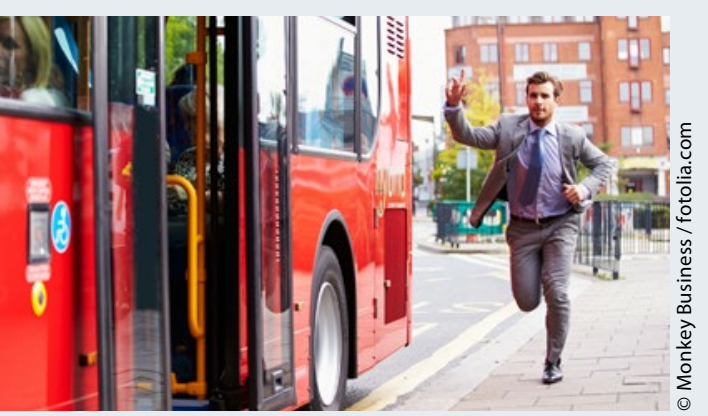

Menschen, die mit Bus oder Bahn zur Arbeit fahren, weisen seltener kardiovaskuläre Risikofaktoren auf als Beschäftigte, die mit dem Auto zur Arbeit fahren, das ergab eine japanische Studie.

Viel körperliche Aktivität beugt Erkrankungen wie Diabetes, Übergewicht und Bluthochdruck vor. Der Grad an Bewegungsaktivität im Alltag und damit der kardiovaskuläre Gesundheitsstatus ist womöglich auch davon abhängig, wie man täglich zum Arbeitsplatz gelangt. Das legen jedenfalls Studiendaten der Gruppe um Dr. Hisako Tsuji aus Osaka nahe, die 2012 die Prävalenz kardiovaskulärer Risikofaktoren bei rund $5.900 \mathrm{Er}$ wachsenen analysiert hat, die den Weg zur Arbeit zu Fuß, mit dem Fahrrad oder mit öffentlichen Verkehrsmitteln (Bus, Bahn) sowie per Auto bewältigten. Bei ihrer für Faktoren wie Alter, Geschlecht oder Rauchen adjustierten Analyse stießen die Untersucher auf Unterschiede.

So war im Vergleich zu Personen, die das Auto nutzten, bei Nutzern von Bus oder Bahn die Wahrscheinlichkeit, dass sie übergewichtig waren, relativ um $44 \%$ geringer. Die Prävalenz von Hypertonie war relativ um $27 \%$ niedriger. Ein Diabetes wurde bei einem um 34\% geringeren Risiko deutlich seltener beobachtet.

Überraschenderweise waren die Prävalenzraten bei Bus- und Bahnfahrern sogar niedriger als bei Personen, die den Weg per Pedes oder Rad zurücklegten. Die Untersucher erklären sich das mit den eventuell längeren Fußwegen, die zwischen Bus- oder Bahnhaltestelle und Arbeitsplatz zu bewältigen waren.

Für die Empfehlung, zur Förderung der Herzgesundheit vom Auto auf Bus oder Bahn umzusteigen, liefert die Studie aber keine triftige Begründung. Wissenschaftlich betrachtet wurden lediglich Assoziationen gezeigt. Ob tatsächlich eine Kausalität zwischen Transportmittel und der Häufigkeit kardiovaskulärer Risikofaktoren besteht, bleibt unklar.

\title{
Duale Plättchenhemmung: Absetzen geschieht oft wegen leichterer Nebenwirkungen
}

\author{
Einer neuen Auswertung der PEGASUS TIMI 54-Studie zufolge setzten viele \\ Postinfarktpatienten Ticagrelor als Bestandteil einer dualen Plättchenhem- \\ mer-Therapie im ersten Jahr aufgrund von relativ gering- bis mäßiggradigen \\ Blutungen und Atembeschwerden ab. Eine bessere Patientenaufklärung \\ könnte dem vorbeugen.
}

$M$ acht es Sinn, bei Postinfarktpatienten die duale PlättchenhemmerTherapie länger als ein Jahr durchzuführen? Zu dieser Frage geben drei Studien Antwort.

\section{Drei Studien geben Antworten}

Eine Post-hoc-Analyse der CharismaStudie fand eine 23\%ige relative Risikoreduktion für weitere atherothrombotische Komplikationen, ohne dass dadurch das Blutungsrisiko signifikant anstieg.

In der im letzten Jahr anlässlich des AHA-Kongresses publizierten prospektiven DAPT-Studie war die Bilanz gemischt: Das Risiko für Atherothrombosen wurde um 1,6\% absolut gesenkt, dasjenige für mittelschwere und schwere Blutungen um 0,9\% absolut erhöht.

\section{Nutzen und Risiko ausgewogen}

Ähnlich war das Ergebnis der im Frühjahr beim Jahreskongress der American College of Cardiology (ACC 2015) publizierten prospektiven PEGASUS TIMI 54-Studie mit 21.162 Postinfarktpatienten, die in den vergangenen ein bis drei Jahren einen Herzinfarkt erlitten hatten und nun für drei Jahre entweder mit Ticagrelor/ASS oder mit ASS allein behandelt wurden.

Auch hier war der Wirksamkeitsendpunkt erreicht worden, die Rate atherothrombotischer Komplikationen sank innerhalb von drei Jahren absolut um $1,2 \%$ von $9 \%$ in der Kontrollgruppe auf 7,8\% in der Ticagrelor/ASS-Gruppe. Gleichzeitig stieg aber die Rate relevanter Blutungen von $1,1 \%$ unter ASS alleine auf 2,3\% bzw. 2,6\% unter der Kombination, je nachdem, ob Ticagrelor mit $60 \mathrm{mg} / \mathrm{d}$ oder $90 \mathrm{mg} / \mathrm{d}$ dosiert worden war.

Wie Dr. Marc P. Bonaca vom Brigham and Women's Hospital in Boston nun beim AHA-Kongress 2015 in Orlando ausführte, ist die Bilanz in der On-Treat-
ment-Analyse etwas günstiger für die duale Thrombozytenaggregationshemmung mit Ticagrelor/ASS: Herztod, Herzinfarkt oder Schlaganfall erlitten $8,4 \%$ der Patienten unter ASS-Monotherapie sowie 6,6\% der Patienten unter der Kombination, ein absolute Differenz von $1,8 \%$.

Pro 1.000 Patienten werden 27 atherothrombotische Komplikationen verhindert, für den Preis von 19 zusätzlichen schweren Blutungen (TIMI major bleedings). Doch auch diese Bilanz verlangt sicher nach einer weiteren Risikostratifizierung, um die Patienten besser zu erkennen, die von der verlängerten dualen Plättchenhemmung tatsächlich profitieren.

\section{Problem Adhärenz}

Ein weiteres Problem ist die Adhärenz. Laut Bonaca haben in der PEGASUS Studie vor allem im ersten Jahr 16\% (90 $\mathrm{mg} / \mathrm{d}$ ) bzw. 13\% (60 mg/d) der Patienten Ticagrelor abgesetzt, im Vergleich zu 6\% in der ASS-Monotherapie-Gruppe. In den Folgejahren setzten dagegen mit 3\% (duale Therapie) bzw. 2,2\% (Monotherapie) jährlich deutlich weniger Patienten die Therapie ab.

Bonaca untersuchte die Absetzgründe. In ca. zwei Drittel der Fälle gaben Nebenwirkungen den Ausschlag und hier vor allem Dyspnoe oder Blutungen. Diese erwiesen sich jedoch in über $85 \%$ der Fälle als gering- bis mäßiggradig. Laut Bonaca sollte deshalb insbesondere im ersten Jahr einer dualen Plättchenhemmung sehr intensiv mit dem Patienten gesprochen werden, um die Adhärenz zu verbessern und einem Absetzen der Medikation vorzubeugen. Dr. med. Dirk Einecke

Literatur: Bonaca MP et al. Long-term use of ticagrelor in patients with prior myocardial infarction. N Engl J Med. 2015;372(19):1791-800. doi: 10.1056/NEJMoa1500857 